\title{
Calibration of Near-Infrared Detectors Using a Wavelength Tunable Light Source
}

\author{
Kinza Maham $^{1} \oplus \cdot$ Anna Vaskuri $^{1,3} \cdot$ Farshid Manoocheri $^{1} \cdot$ Erkki Ikonen $^{1,2}$ \\ Received: 15 October 2019 / Accepted: 1 February 2020 / Published online: 17 February 2020 \\ (c) The Author(s) 2020
}

\begin{abstract}
This paper presents the spectral responsivity calibrations of two indium gallium arsenide (InGaAs) and one germanium based near-infrared photovoltaic detectors using a wavelength tunable laser source based on a supercontinuum laser developed at the Metrology Research Institute, Aalto University. The setup consists of a supercontinuum laser based on a photonic crystal fiber as the light source, a laser line tunable filter, and coupling optics. These responsivity calibrations are performed against a pyroelectric radiometer over a wide spectral range of 800-2000 $\mathrm{nm}$. Our wavelength tunable laser source has a high spectral power up to $2.5 \mathrm{~mW}$ with a narrow spectral full-width-at-half-maximum of $3 \mathrm{~nm}$ at a wavelength of $1100 \mathrm{~nm}$. Despite the sharp spectral intensity variations, no artifacts are observed in the spectral responsivities of the detectors. Comparison of the spectral responsivities of the InGaAs detectors measured using the wavelength tunable laser and the earlier calibrations performed at the Metrology Research Institute in 2010 and 2016, shows that the higher spectral power of wavelength tunable light source decreases the expanded uncertainty from approximately $4 \%$ to $2.2-2.6 \%$ over the spectral range of $820-1600 \mathrm{~nm}$. Temperature dependence of the spectral responsivities near the band gap edges are also measured and analysed.
\end{abstract}

Keywords Near-infrared detectors $\cdot$ Spectral responsivity $\cdot$ Supercontinuum laser $\cdot$ InGaAs $\cdot$ Ge

\section{Introduction}

Absolute calibration of optical detectors is important for the reliability of optical characterization methods and, therefore, has significant applications in optics and metrology. Absolute cryogenic radiometer (ACR), based on electrical substitution, has been used as a primary standard instrument in optical power calibrations of detectors at many metrology institutes since 1980's [1-3]. However, when ACRs are coupled with traditional lamp-monochromators, limited radiant flux of about $100 \mathrm{nW} / \mathrm{nm}$ is observed even at moderate spectral resolution, as the lamp-monochromator systems

Kinza Maham

kinza.maham@aalto.fi

1 Metrology Research Institute, Aalto University, P.O. Box 15500, FI-00076 Aalto, Finland

2 VTT MIKES, P.O. Box 1000, FI-02044 VTT Espoo, Finland

3 Present Address: National Institute of Standards and Technology, Boulder, CO, USA have finite etendue [4]. To address this shortcoming, several wavelength tunable laser source setups based on dye, Ti:Sapphire, or supercontinuum laser have been explored mainly in the visible range [5-12].

Supercontinuum (SC) laser is a source of broad, coherent, and continuous wave white light emission over the broad spectral range from 350 to $2500 \mathrm{~nm}$. SC lasers are based on a photonic crystal fiber (PCF) with a range of available pump powers. These lasers also have three orders of magnitude higher radiant flux of $5 \mathrm{~mW} / \mathrm{nm}$ in comparison to typical lamp-monochromators [4]. Therefore, SC lasers can be employed in many fields including optical metrology as they allow measurements with low etendue, high power, and high spectral resolution.

In this paper, we present a newly developed monochromatic light source based on a SC laser for the spectral responsivity calibrations of two indium gallium arsenide (InGaAs) and one germanium (Ge) based photovoltaic near-infrared detectors at the spectral range of $800 \mathrm{~nm}-2000 \mathrm{~nm}$. A laser line tunable filter (LLTF) is used to obtain a collimated wavelength tunable light beam from 
the supercontinuum laser. These spectral responsivity calibrations are performed against a calibrated pyroelectric radiometer which has a spectrally flat responsivity.

The setup introduced in this paper is automated using custom-made LabVIEW software, which increases the detector alignment accuracy and repeatability of the calibration measurements. Furthermore, our wavelength tunable laser source developed provides narrower spectral bandwidths with higher optical power output compared to traditional lamp-based monochromators. Effects of sharp spectral features in the source spectrum are analysed and found to be insignificant. Our setup decreases the expanded uncertainty in the spectral responsivity calibrations to approximately $2.3 \%$.

\section{Optical setup and measurement sequence}

The optical setup of the wavelength tunable laser source developed for the spectral responsivity calibrations of the photovoltaic detectors is presented in Fig. 1. A supercontinuum laser based on PCF is the source of optical radiation in our setup. The low etendue of the supercontinuum laser enables accurate collimation. We have used a laser line tunable filter (LLTF) in order to obtain wavelength tunable light beam from the supercontinuum laser. LLTF is based on volume holographic Bragg grating made of silver halide glass, which is a non-hygroscopic material and is transparent between 400 and $2500 \mathrm{~nm}$. The input beam is diffracted by the gratings present in the LLTF. Figure 2 shows a simplified design of the LLTF.

In order to select the diffracted wavelength of light, the angle of the grating is adjusted to meet the Bragg's condition $\lambda_{\mathrm{b}}=2 n_{\mathrm{o}} \Lambda \cos (\Theta+\phi)$, where $\lambda_{\mathrm{b}}$ is the Bragg wavelength, $\Lambda$ is the period of the grating, $n_{\mathrm{o}}$ is the refractive index of the photo-thermo-refractive glass, $\Theta$ is the angle between incident light and normal of the grating surface, and $\phi$ is the inclination of Bragg plane which is defined as the angle between the normal of the grating surface and the grating vector [14]. If the light beam does not satisfy the Bragg condition, it passes through undiffracted. Only a particular narrow wavelength range fulfills Bragg condition and

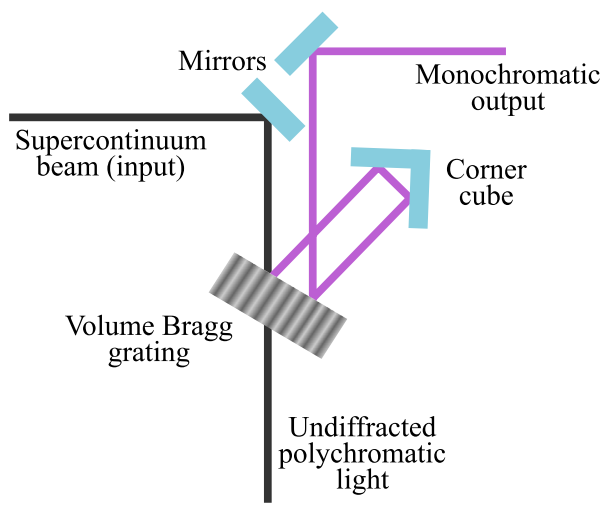

Fig. 2 Design of the laser line tuneable filter (LLTF) based on a volume Bragg grating. The figure is modified from [13]

interferes constructively with the refractive index modulation. The diffracted light is reflected on a second region of the Bragg grating by a corner cube to achieve double diffracted monochromatic output beam.

The LLTF in use consists of two gratings: grating \#1 is dedicated to $1000-1700 \mathrm{~nm}$, while grating \#2 is dedicated to $1700-2000 \mathrm{~nm}$ [15]. The wavelength scale of the LLTF is characterized using a scanning spectroradiometer and wavelength shifts as presented in Table 1, are observed and corrected from the final spectral responsivity calibration results.

The standard uncertainty of $\Delta \lambda$ is estimated by deviations from a smooth fitting curve and it also includes the uncertainty contribution from the calibration of the spectroradiometer. A standard uncertainty of $0.03 \%$ is present in the spectral responsivities of the photovoltaic detectors due to the wavelength of the laser after correction.

The spectroradiometer used for the characterization of LLTF is calibrated using mercury $(\mathrm{Hg})$ and argon (Ar) calibration pen lamps. These calibration lamps produce intense fundamental spectral lines from the excitation of $\mathrm{Hg}$ vapor and Ar that do not shift with the temperature and time.

The collimated light from LLTF is coupled into a multimode fiber having a numerical aperture (NA) of 0.22 . To ensure optimal coupling of the beam into the fiber, a lens is placed in the fiberoptic module connected at the output port of the LLTF. The multimode fiber terminates into a
Fig. 1 Wavelength tunable filter light source setup, based on a supercontinuum laser and a laser line tunable filter (LLTF) used for measuring the spectral responsivity of photovoltaic detectors

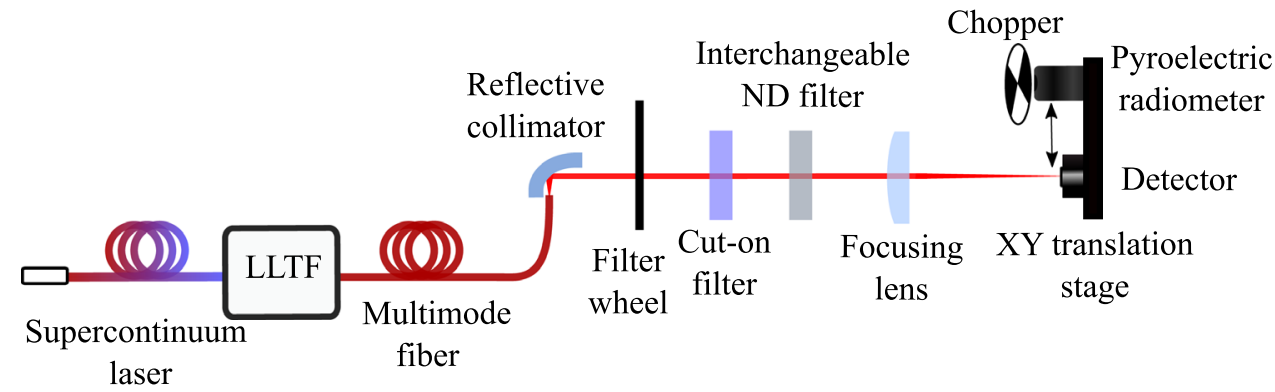


Table 1 Wavelength shift $\Delta \lambda$ between the observed wavelength and the set wavelength of the LLTF with its standard uncertainty

\begin{tabular}{llll}
\hline Observed $\lambda / \mathrm{nm}$ & Set $\lambda / \mathrm{nm}$ & $\Delta \lambda / \mathrm{nm}$ & $\begin{array}{l}\text { Standard } \\
\text { uncertainty/ } \\
\mathrm{nm}\end{array}$ \\
\hline 997.06 & 1000 & -2.94 & 0.15 \\
1097.33 & 1100 & -2.67 & 0.15 \\
1197.15 & 1200 & -2.85 & 0.15 \\
1297.22 & 1300 & -2.78 & 0.15 \\
1397.23 & 1400 & -2.77 & 0.15 \\
1497.37 & 1500 & -2.63 & 0.15 \\
1597.66 & 1600 & -2.34 & 0.15 \\
1702.23 & 1700 & 2.23 & 0.25 \\
1801.19 & 1800 & 1.19 & 0.25 \\
1900.74 & 1900 & 0.74 & 0.25 \\
1999.28 & 2000 & -0.72 & 0.25 \\
\hline
\end{tabular}

reflective collimator, based on a $90^{\circ}$ off-axis parabolic mirror. A motorized filter wheel, controlled by LabVIEW software, is used to measure dark and signal voltages for each wavelength [16]. We observed that the LLTF produces strong second and third harmonics in the visible wavelength range. Therefore, a cut-on filter with up to $99.9 \%$ attenuation of all the wavelengths below $800 \mathrm{~nm}$ was used for filtering the harmonics.

The laser setup has an average power of up to $0.3 \mathrm{~mW}$ within a $3 \mathrm{~nm}$ band from $800-2000 \mathrm{~nm}$. In order to avoid saturation of the detectors, an interchangeable neutral density filter with the optical density of 0.5 is used to attenuate the optical power of the beam. The setup produces a collimated uniform beam with a narrow diameter of approximately $2 \mathrm{~mm}$. The setup is further automated by mounting the photovoltaic and reference detectors on a high-resolution $X Y$ translation stage, which allows us to obtain the pyroelectric reference signal and detector voltage signal with high precision for every wavelength.

The pyroelectric radiometer is a thermal detector, with a lithium tantalate pyroelectric probe, which operates on the principle of temperature change induced temporal voltage difference $[17,18]$. We use it as a reference detector because it has a flat spectral responsivity. Our pyroelectric radiometer runs at a chopping frequency of $30 \mathrm{~Hz}$. The reference detector is calibrated against a silicon trap detector at a wavelength of $647 \mathrm{~nm}$. The calibration of the pyroelectric radiometer and its spectral flatness accounts for a standard uncertainty of $1 \%$.

The spectral responsivities of detectors are measured from $800-2000 \mathrm{~nm}$ with an interval of $0.5 \mathrm{~nm}$. A delay of $50 \mathrm{~ms}$ is added before each reading, to ensure the detectors have stabilized. A current-to-voltage converter is used with the photovoltaic detectors. The measurement sequence of the software records four sets of voltages for each wavelength:

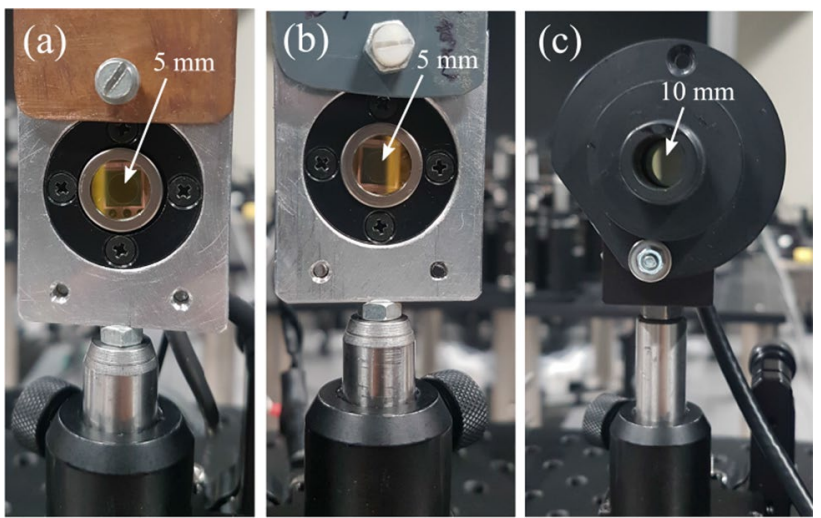

Fig. 3 Detectors studied in this work: InGaAs \#1 detector with an aperture of $5 \mathrm{~mm}$ (a), InGaAs \#2 with an aperture of $5 \mathrm{~mm}(\mathbf{b})$, and Ge detector with an aperture of $10 \mathrm{~mm}(\mathbf{c})$

reference signal voltage $V_{\mathrm{p} \text {, signal }}$, dark reference voltage $V_{\mathrm{p} \text {, dark }}$, signal voltage of the detector $V_{\text {signal }}$ and dark voltage of the detector $V_{\text {dark }}$. For each wavelength between 800 and $2000 \mathrm{~nm}$ in the calibration, the spectral responsivity (A/W) is calculated as

$R=\frac{I}{P_{\text {ref }}}$,

where $I$ is measured photocurrent generated by the photovoltaic detector under test and $P_{\text {ref }}$ is the reference power of pyroelectric radiometer. Current of the photodetectors is calculated from the measured voltages as, $I=\left(V_{\text {signal }}-V_{\text {dark }}\right) \cdot \frac{1}{G_{\mathrm{CVC}}}$, where $G_{\mathrm{CVC}}$ is the calibrated gain of a current-to-voltage converter (CVC). In order to calculate the reference power, equation $P_{\text {ref }}=c_{\mathrm{p}} \cdot\left(V_{\mathrm{p} \text {, signal }}-V_{\mathrm{p} \text {, dark }}\right)$ is used, where the correction factor $c_{\mathrm{p}}$ includes the correction from the absolute calibration of the pyroelectric radiometer.

\section{Results}

Figure 3 is a photograph of the InGaAs \#1, InGaAs \#2, and Ge photovoltaic detectors calibrated against a pyroelectric radiometer. These detectors have wavelength dependent spectral responsivities, fast response times, and high sensitivities [19].

The InGaAs detectors shown in Fig. 3 have an aperture diameter of $5 \mathrm{~mm}$ and operate over the spectral range of $900-1680 \mathrm{~nm}$, with a band gap edge near $1750 \mathrm{~nm}$. The Ge photovoltaic detector has an aperture diameter of $10 \mathrm{~mm}$. It has a high spectral responsivity over a spectral range of approximately $750-1850 \mathrm{~nm}$ with a band gap edge at approximately $1650 \mathrm{~nm}$. In this work, we have defined the 
usable spectral range as a region where the spectral responsivity exceeds $10 \%$ from the maximum response.

\subsection{Spectral responsivities of InGaAs detectors}

The spectral responsivity calibrations of InGaAs detectors were performed at three different temperatures of $273.15 \mathrm{~K}$, $283.15 \mathrm{~K}$, and $297.15 \mathrm{~K}$. Figures $4 \mathrm{a}$ and $5 \mathrm{a}$ show the spectral responsivities of InGaAs \#1 and InGaAs \#2 detectors over the spectral range of 800-2000 $\mathrm{nm}$. The detectors were cooled down with their internal temperature controllers. The incident optical power measured using the pyroelectric radiometer is also shown as the black dotted curve.

As can be seen in Figs. 4a and 5a, the beam power of the wavelength tunable laser varies significantly across the spectral range with a maximum power of approximately $0.7 \mathrm{~mW}$ at $1100 \mathrm{~nm}$ when the neutral density filter is used. Despite the sharp peaks and irregularities in the spectrum of the supercontinuum laser, no significant deviations are present in the spectral responsivities. To study this, first-order
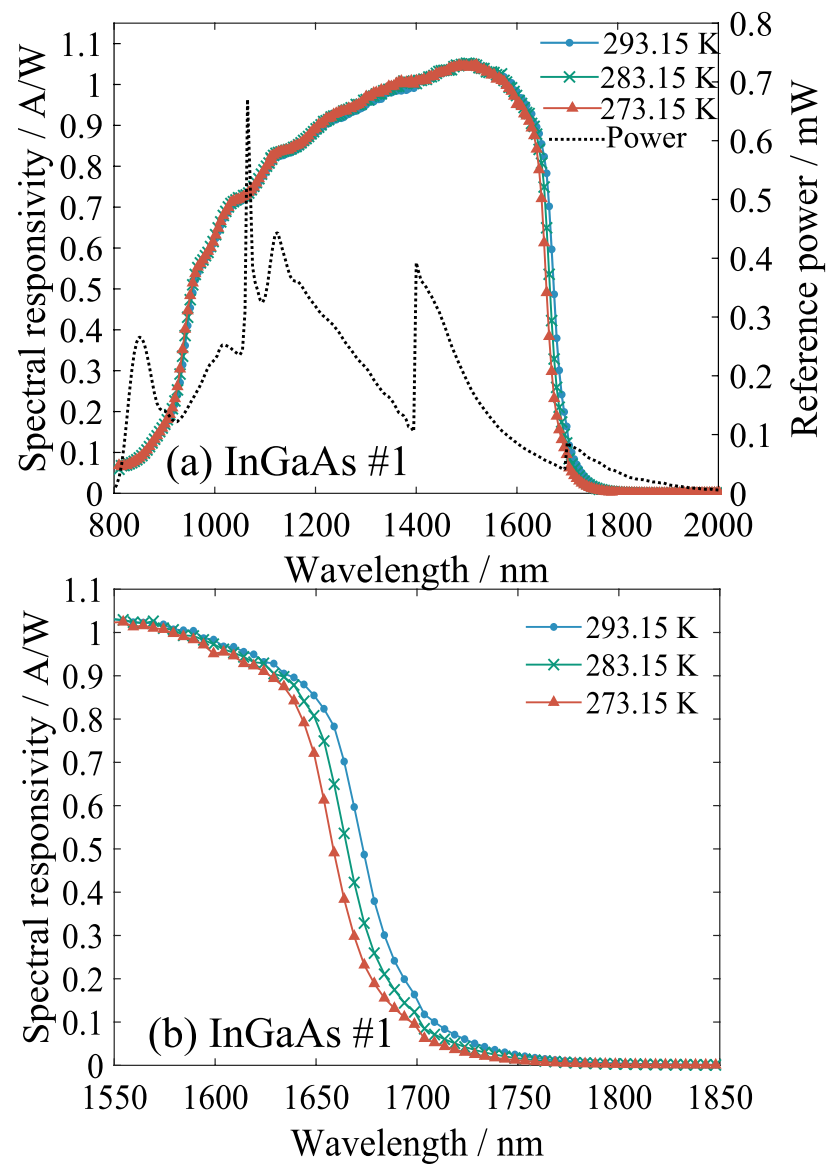

Fig. 4 Spectral responsivity of the InGaAs \#1 measured with the wavelength tunable laser source over the spectral range of 800-2000 nm along with the optical reference power (a). Close-up of spectral responsivity of InGaAs \#1 near the band gap edge (b)

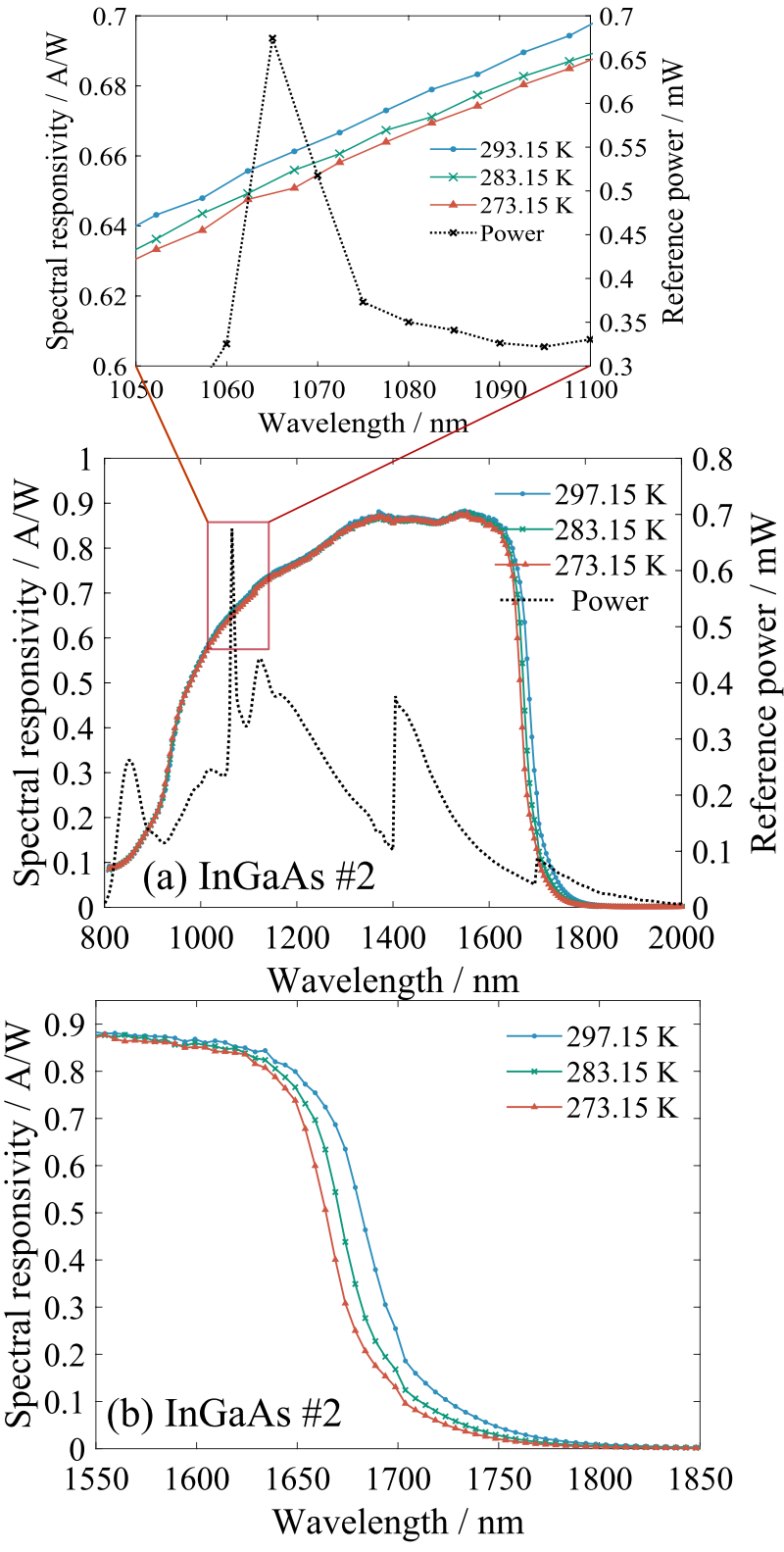

Fig. 5 Spectral responsivity of the InGaAs \#2 measured with the wavelength tunable laser source over the spectral range of 800-2000 nm along with the optical reference power (a). Details of the responsivity are magnified close to the peak power. Close-up of spectral responsivity of InGaAs \#2 near the band gap edge (b)

polynomials were fitted to the spectral responsivities of InGaAs \#2 between 1050 and $1100 \mathrm{~nm}$, close to the peak power. The fitting residuals have relative standard deviations smaller than $0.10 \%$.

The two InGaAs detectors have similar spectral responsivity curves, however, higher oscillations are observed in the spectral responsivity of detector \#1. The junction temperature is also an important factor for these detectors since the band gap edge of the spectral responsivity shifts towards shorter wavelengths with decreasing temperature. Figures $4 \mathrm{~b}$ 
and $5 \mathrm{~b}$ show close-ups of the spectral responsivities of the InGaAs detectors near the band gap edge.

The spectral responsivity calibrations obtained in this work were compared to the older calibrations measured with a reference spectrometer at the Metrology Research Institute, Aalto University [20]. The calibration of InGaAs \#1 against the pyroelectric radiometer was compared with the previous calibration performed in 2016 at $283.15 \mathrm{~K}$ with an expanded uncertainty of $4 \%$. The comparison plot of InGaAs \#1 is presented in Fig. 6a. The spectral responsivity of InGaAs \#2 was calibrated in 2010 at the room temperature of $297.65 \mathrm{~K}$ $\pm 1.5 \mathrm{~K}$ with an expanded uncertainty of $4 \%$. The spectral responsivity of InGaAs \#2 calibrated in 2010 is presented in Fig. $6 \mathrm{~b}$ along with the responsivities measured with the wavelength tunable laser source, against a pyroelectric radiometer.

Detector calibrations against the pyroelectric radiometer have an expanded uncertainty of $2.2-2.6 \%$ over the spectral range of 820 and $1600 \mathrm{~nm}$ depending on the wavelength. The slight difference in our calibration and older reference calibration can be attributed to the uncertainty present in both calibrations. The ageing of the detectors can also be one reason for the different spectral responsivities of InGaAs detectors.
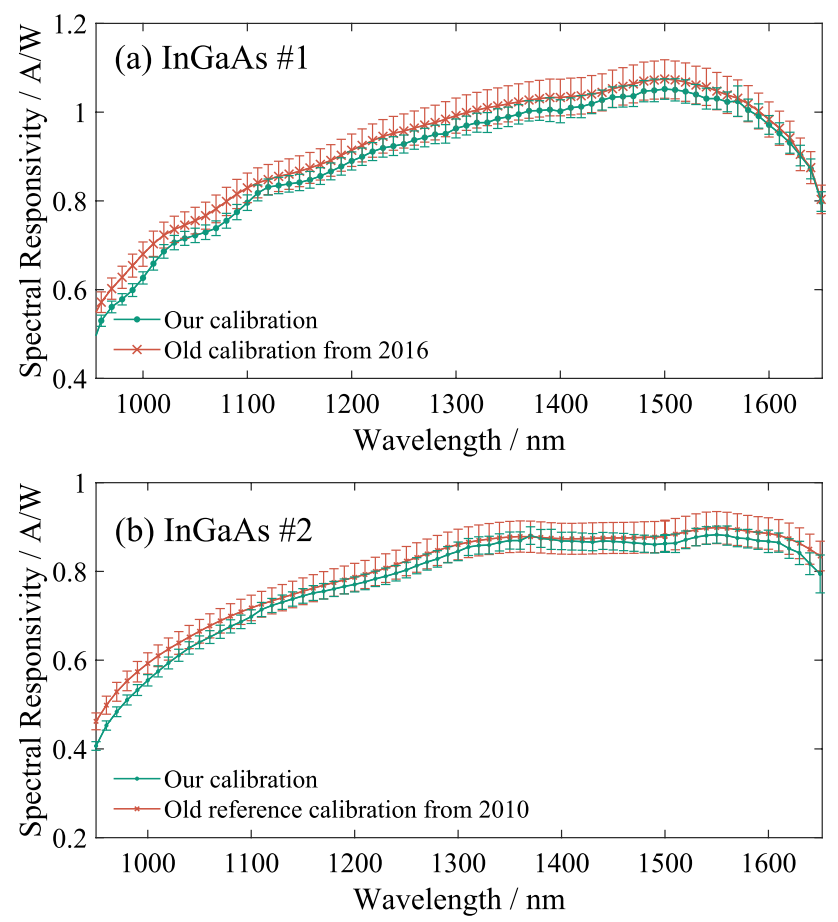

Fig. 6 Comparison of spectral responsivities measured with the wavelength tunable laser source and the reference pyroelectric radiometer with older calibration of InGaAs detectors \#1 (a) and \#2 (b). The uncertainty bars indicate expanded uncertainties $(k=2)$ in the spectral responsivities

\subsection{Spectral responsivity of a Ge detector}

Figure 7 shows the spectral responsivity of the Ge detector and reference power recorded using the pyroelectric radiometer.

Unlike InGaAs, this Ge detector does not have a cooling option; therefore, it was calibrated at room temperature that was $298.15 \mathrm{~K}$ during the measurements. The Ge detector has low shunt resistance and in turn significant dark current, which highly depends on the temperature, hence, it is very sensitive to the ambient temperature [21]. The Ge photodiode also has an ageing rate of approximately $0.5 \%$ per year within a spectral range of $1100 \mathrm{~nm}-1800 \mathrm{~nm}$ as studied by Stock [22] and Lamminpää et al. [23].

\subsection{Uncertainty budget}

Table 2 gives the uncertainty budget of the detector responsivity calibrations. The pyroelectric radiometer has been calibrated against a calibrated silicon trap detector. The calibration of the pyroelectric radiometer and its spectral flatness accounts for a standard uncertainty of $1 \%$. The uncertainty due to spatial uniformity of the pyroelectric detector is also included in the reference detector uncertainty. The external chopper used with the pyroelectric radiometer produces a standard uncertainty of $0.42 \%$ due to the manual adjustment of its phase.

A standard uncertainty of $0.13 \%$ is present in the setup due to the alignment of detectors. This uncertainty is was determined by tilting the detector by $\pm 1^{\circ}$ and measuring the relative changes in the detected response. The wavelength of the laser contributes with a standard uncertainty of $0.03 \%$. The spatial nonuniformity of the interchangeable attenuating neutral density filter also contributes with an

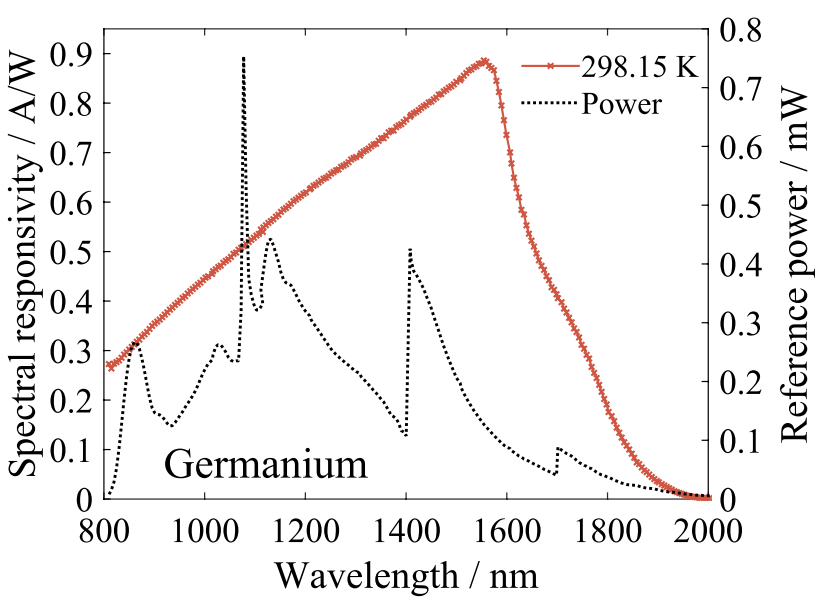

Fig. 7 Spectral responsivity of the Ge detector measured with the wavelength tunable laser source at room temperature along with the reference optical power measured using a pyroelectric radiometer 
Table 2 Uncertainty budget of the spectral responsivity of detectors at the wavelength range from 820 to $1600 \mathrm{~nm}$

\begin{tabular}{ll}
\hline Source of uncertainty & Uncertainty/\% \\
\hline Reference detector & 1.00 \\
Phase resolution of chopper & 0.42 \\
Alignment of detector & 0.13 \\
Wavelength of laser & 0.03 \\
Spatial uniformity of neutral density filter & 0.27 \\
Average repeatability of measurement & 0.26 \\
Calibration of the current-to-voltage converter & 0.001 \\
Calibration of the multimeter & 0.001 \\
Combined standard uncertainty $(k=1)$ & 1.15 \\
Expanded uncertainty $(k=2)$ & 2.30 \\
\hline
\end{tabular}

The pyroelectric radiometer was used as a reference detector

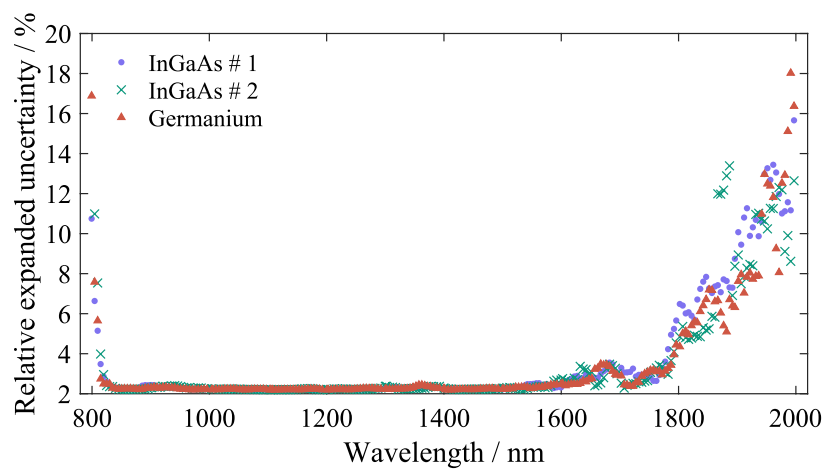

Fig. 8 Relative expanded uncertainties in the spectral responsivities of the detectors studied from $800-2000 \mathrm{~nm}$

additional standard uncertainty of $0.27 \%$. Another source of uncertainty is the repeatability of the results. The spectral responsivity measurements were repeated three times for every wavelength, and their average and standard deviation were calculated for each wavelength. The average uncertainty calculated due to the repetition of measurements over the spectral range of $820-1600 \mathrm{~nm}$ is $0.26 \%$.

Expanded uncertainties for the three detectors are shown in Fig. 8. InGaAs detectors have high spectral responsivity over the spectral range of $900 \mathrm{~nm}-1680 \mathrm{~nm}$ while the Ge detectors have high spectral responsivity between $850-1650 \mathrm{~nm}$. It can also be observed from Figs. 4, 5, and 7 , that the signal power of wavelength tunable laser source is high only between $820-1600 \mathrm{~nm}$. Therefore, the expanded uncertainty at these wavelengths is relatively low and starts increasing near at the band gap edges of the detectors. In addition, although our reference pyroelectric radiometer response is supposed to be linear with optical power, we noticed that it behaves nonlinearly below $20 \mu \mathrm{W}$. The nonlinerity error is $1.7 \%$ at $10 \mu \mathrm{W}$ level and $6.1 \%$ at $5 \mu \mathrm{W}$ level. In practice, this effect increases the spectral responsivity uncertainties at the spectral range of 1800-2000 nm, where the reference power decreases below $20 \mu \mathrm{W}$ (Fig. 3a). The spectral range of $800-1800 \mathrm{~nm}$ is unaffected by this effect. In these spectral responsivity calibrations, the reference detector causes the highest uncertainty. The expected uncertainty $(k=2)$ can be reduced to $0.5 \%$ by using a different reference detector with low uncertainty.

\section{Summary}

Two InGaAs and one Ge photovoltaic detectors were calibrated using a portable wavelength tunable light source based on a supercontinuum laser. The setup comprises of a supercontinuum laser as a light source, LLTF, and coupling optics. The setup operates over a spectral range of 800-2000 nm with a maximum power of approximately $0.7 \mathrm{~mW}$ and fullwidth-at-half-maximum of $3 \mathrm{~nm}$ at a wavelength of $1100 \mathrm{~nm}$. A calibrated pyroelectric radiometer is used as a reference detector due to its flat spectral responsivity.

The spectral responsivities measured were compared to the older calibrations performed in 2010 and 2016, at the Metrology Research Institute, Aalto University. With new setup the expanded uncertainties in the spectral responsivity calibrations decreased from approximately $4 \%$ to $2.2-2.6 \%$, at the spectral range of $820-1600 \mathrm{~nm}$. Expanded uncertainties became higher, up to $18 \%$, below $820 \mathrm{~nm}$ and above $1600 \mathrm{~nm}$ due to the decreasing incident laser power and decreased responsivity near the band gap edges of the photodetectors.

Acknowledgements Open access funding provided by Aalto University.

Open Access This article is licensed under a Creative Commons Attribution 4.0 International License, which permits use, sharing, adaptation, distribution and reproduction in any medium or format, as long as you give appropriate credit to the original author(s) and the source, provide a link to the Creative Commons licence, and indicate if changes were made. The images or other third party material in this article are included in the article's Creative Commons licence, unless indicated otherwise in a credit line to the material. If material is not included in the article's Creative Commons licence and your intended use is not permitted by statutory regulation or exceeds the permitted use, you will need to obtain permission directly from the copyright holder. To view a copy of this licence, visit http://creativecommons.org/licenses/by/4.0/.

\section{References}

1. Martin, J.E., Fox, N.P., Key, P.J.: A cryogenic radiometer for absolute radiometric measurements. Metrologia 21, 147-155 (1985)

2. Varpula, T., Seppä, H., Saari, J.M.: Optical power calibrator based on a stabilized green He-Ne laser and a cryogenic absolute radiometer. IEEE Trans. Instrum. Meas. 38, 558-564 (1989)

3. Foukal, P.V., Hoyt, C., Kochling, H., Miller, P.: Cryogenic absolute radiometers as laboratory irradiance standards, remote 
sensing detectors, and pyroheliometer. Appl. Opt. 29, 988-993 (1990)

4. Woodward, J.T., Smith, A.W., Jenkins, C.A., Lin, C., Brown, S.W., Lykke, K.R.: Supercontinuum sources for metrology. Metrologia 46, S277-S282 (2009)

5. Anderson, V.E., Fox, N.P., Nettleton, D.H.: Highly stable, monochromatic and tunable optical radiation source and its application to high accuracy spectrophotometry. Appl. Opt. 31, 536-545 (1992)

6. Noorma, M., Toivanen, P., Manoocheri, F., Ikonen, E.: Characterization of filter radiometers with a wavelength-tunable laser source. Metrologia 40, S220-S223 (2003)

7. Ahtee, V., Brown, S.W., Larason, T.C., Lykke, K.R., Ikonen, E., Noorma, M.: Comparison of absolute spectral irradiance responsivity measurement techniques using wavelength-tunable lasers. Appl. Opt. 46, 4228-4236 (2007)

8. Zarobila, C.J., Patrick, H.J.: Supercontinuum fiber laser source for reflectance calibrations in remote sensing. Proc. SPIE 7807, B-1-16 (2010)

9. Ceolato, R., Riviere, N., Hespel, L.: Reflectances from a supercontinuum laser-based instrument: hyperspectral, polarimetric and angular measurements. Opt. Express 20, 29413-29425 (2012)

10. Schuster, M., Nevas, S., Sperling, A., Völker, S.: Spectral calibration of radiometric detectors using tunable laser sources. Appl. Opt. 51, 1950-1961 (2012)

11. Levick, A.P., Greenwell, C.L., Ireland, J., Woolliams, E.R., Goodman, T.M., Bialek, A., Fox, N.P.: Spectral radiance source based on supercontinuum laser and wavelength tunable bandpass filter: the spectrally tunable absolute irradiance and radiance source. Appl. Opt. 53, 3508-3519 (2014)

12. Bünger, L., Taubert, R. D., Anhalt, K.: Spectrally resolved radiometric characterisation and calibration using a supercontinuum laser in the NIR. In: Proc. IRS ${ }^{2}$, pp. 895-898 (2015)
13. Marcet, S., Verhaegen, M., Blais-Ouellette, S., Martel, R.: Raman spectroscopy hyperspectral imager based on bragg tunable filter. Proc. SPIE 8231, 82310U1-8 (2012)

14. Gagnon, D., Dion-Bertrand, L.I.: Widely tunable filter technology and measurement of critical specifications. White Paper. Photon Etc., pp. 1-13 (2015)

15. Photon Etc.: Laser Line Tunable Filter (LLTF) contrast ${ }^{\mathrm{TM}}$. Instruction Manual, pp. 21-22 (2017)

16. Vaskuri, A.: Multi-Wavelength setup based on lasers for characterizing optical detectors and materials. Master's Thesis, Aalto University (2014)

17. Hamilton, C.A., Day, G.W., Phelan, R.J.: An electrically calibrated pyroelectric radiometer system. Technical Note 678, Electromagnetics Division Institute for Basic Standards, pp. 2-10. National Bureau of Standards, CO, USA (1976)

18. Laser Probe Inc.: RkP-575 pyroelectric power probe: Datasheet, NY, USA

19. Hamamatsu: Characteristics and use of infrared detectors. Technical Information, Hamamatsu Photonics K. K., Solid State Division, Japan (2011)

20. Manoocheri, F., Kärhä, P., Palva, L., Toivanen, P., Haapalinna, A., Ikonen, E.: Characterisation of optical detectors using highaccuracy instruments. Anal. Chim. Acta. 380, 327-337 (1999)

21. Eppeldauer, G.P.: Electronic characteristics of Ge and InGaAs radiometers. Proc. SPIE 3061, 833-838 (1997)

22. Stock, K.D.: Internal quantum efficiency of Ge photodiodes. Appl. Opt. 27, 12-14 (1988)

23. Lamminpää, A., Noorma, M., Hyyppä, T., Manoocheri, F., Kärhä, P., Ikonen, E.: Characterization of germanium photodiodes and trap detector. Meas. Sci. Technol. 17, 908-912 (2006)

Publisher's Note Springer Nature remains neutral with regard to jurisdictional claims in published maps and institutional affiliations. 